\title{
Optical Hydrogen Sensing with Tungsten Oxide Photonic Crystals
}

\author{
Sabrina Amrehn, Xia Wu, Thorsten Wagner \\ Paderborn University, Warburger Straße 100, 33098 Paderborn, Germany \\ sabrina.amrehn@uni-paderborn.de
}

\begin{abstract}
A hydrogen sensor with optical read out based on a photonic crystal is presented. The position of the photonic band gap of a tungsten oxide inverse opal (loaded with platinum) is used as an optical sensor signal to detect hydrogen. To explain the sensor behavior, the mechanism of intercalation of hydrogen into the tungsten oxide lattice, the formation of tungsten bronze, and the back reactions are investigated. This reactions cause a refractive index change of the solid phase and so the photonic band gap shifts. The photonic band gap is, besides others, determined by the refractive index contrast of the two periodically arranged materials, which form the photonic crystal. Also cross-sensitivities for typical gases, like methane, carbon monoxide and humidity are shown. Due to the specific intercalation mechanism for hydrogen the selectivity of the presented system is high.
\end{abstract}

Key words: photonic crystals, hydrogen sensing, optical sensor, tungsten oxide, inverse opal

\section{Photonic Crystals}

The color impression of photonic crystals is caused by their structure. Two materials with different refractive indices are periodically arranged with a periodicity in the order of the wavelength of visible light (see figure 1). Due to this periodic arrangement, light of a certain wavelength range is reflected at this structure. This wavelength range is called the photonic band gap. The position of the photonic band gap is determined, beside others, by the refractive index contrast of the two materials, the photonic crystal consist of. If the refractive index contrast changes, also the band gap position changes. [1]

\section{Gas Sensing with Photonic Crystals}

Metal oxide photonic crystals can be utilized as transducers for gas sensing, because metal oxides change their refractive indices due to gas reactions. So the photonic band gap position of a metal oxide photonic crystal is correlated to gas concentrations. The band gap shift can be detected optically and a remote readout is possible. Because of this, gas sensing in harsh environments is possible with this setup. Only the transducer itself has to stay in contact with the gas atmosphere. The packaging technology in the gas atmosphere, which causes normally the majority of the stability problems of gas sensors in harsh environments, can be substantially reduced.

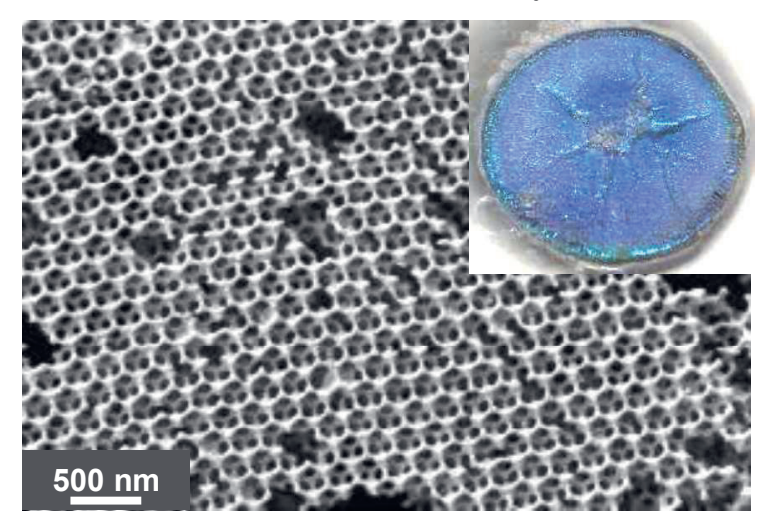

Fig. 1. Photograph (inset) and electron microscope image of a tungsten oxide inverse opal, used for hydrogen sensing.

To monitor gases with photonic crystals, their reflectance spectrum is measured. Afterwards the peak position of the reflectance peak has to be determined as the sensor signal. For fitting the reflectance peak, the Fano resonance model can be used. [2] It considers the Bragg and the Mie diffraction at the nanostructures. [3]

\section{Hydrogen Sensing}

To detect hydrogen with photonic crystal, tungsten oxide inverse opals loaded with platinum were used (figure 1). The characteristics (figure 2) show a correlation 
between the peak position and the gas concentration. The hydrogen intercalates into the tungsten oxide lattice and causes a change in the refractive index. This leads to the photonic band gap shift.

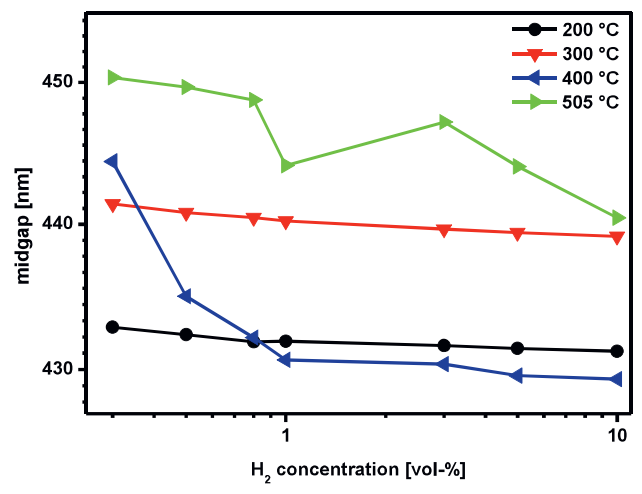

Fig. 2. Peak position of the reflectance peak of the tungsten oxide inverse opal depending on the hydrogen concentration in a log-log-plot. [4]

The response to hydrogen is fast, e.g. t90 $_{90}=0.7 \mathrm{~s}$ for $5 \%$ hydrogen at $200{ }^{\circ} \mathrm{C}$. Up to $300{ }^{\circ} \mathrm{C}$ the characteristics show an exponential behavior. At $400{ }^{\circ} \mathrm{C}$ and above, this changes. So up to $300{ }^{\circ} \mathrm{C}$ the sensor response is dominated by the surface reaction of hydrogen with the inverse opal. Above the back reaction dominates the sensor response. More details about this mechanisms will be shown. [4]

The cross-sensitivities to methane, carbon monoxide and humidity are negligible (methane shown in figure 3 ).

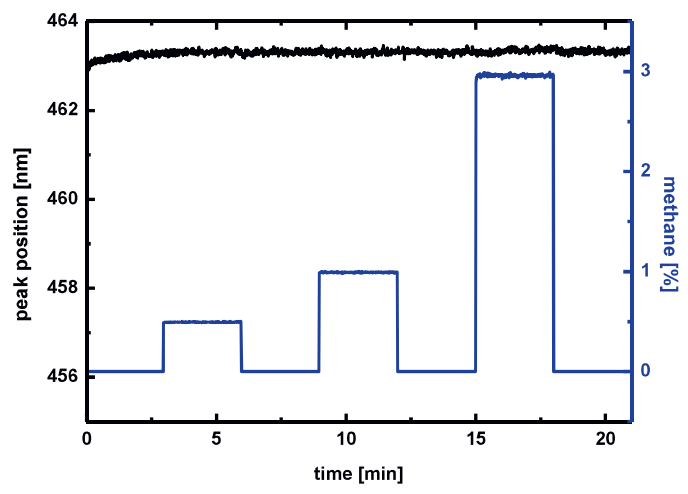

Fig. 3. Peak position of the reflectance peak of the tungsten oxide inverse opal depending on the methane concentration at $400^{\circ} \mathrm{C}$ in dry nitrogen (100 mL/min).

This is due to the mechanism, which is based on the selective intercalation of the target species (hydrogen) into the tungsten oxide lattice. Surface reactions of tungsten oxide with other gases have a much lower effect on the refractive index and the photonic band gap, respectively.

\section{Conclusion}

The shown hydrogen sensor based on tungsten oxide inverse opals can be used to detect hydrogen from $3000 \mathrm{ppm}$ to $10 \%$ up to $500{ }^{\circ} \mathrm{C}$. The fast response and the remote readout offer a wide range of applications also in harsh environments. The negligible cross-sensitivities to methane, carbon monoxide and humidity are related to the hydrogen intercalation mechanism.

\section{References}

[1] J. D. Joannopplus, P. R. Villeneuve, S. Fan, Photonich crytals: putting a new twist on light, Nature 386, 143-149 (1997); doi: $10.1038 / 386143 a 0$

[2] U. Fano, Effects of Configuration Interaction on Intensities and Phase Shifts, Physical Review 124, 1866-1878 (1961); doi: 10.1103/PhysRev.124.1866

[3] M. Rybin, A. Khanikaev, M. Inoue, K. Samusev, M. Steel, G. Yushin, M. Limonov, Fano Resonance between Mie and Bragg Scattering in Photonic Crystals, Physical Review Letters 103, 023901 (2009); doi: 10.1103/PhysRevLett.103.023901

[4] S. Amrehn, X. Wu, T. Wagner, Tungsten Oxide Photonic Crystals as Optical Transducer for Gas Sensing, ACS Sensors 3, 191-199 (2018); doi: 10.1021/acssensors.7b00845 